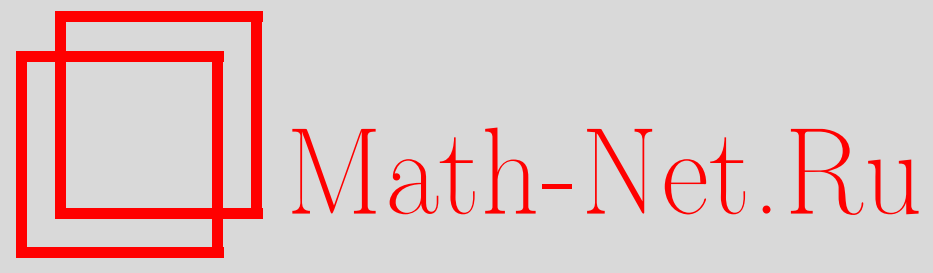

А. М. Ильясов, Г. Т. Булгакова, Квазиодномерная модель гиперболического типа гидроразрыва пласта, Вестн. Сам. гос. техн. ун-та. Сер. Физ.мат. науки, 2016, номер 4, 739-754

DOI: https://doi.org/10.14498/vsgtu1522

Использование Общероссийского математического портала MathNet.Ru подразумевает, что вы прочитали и согласны с пользовательским соглашением

http://www . mathnet.ru/rus/agreement

Параметры загрузки:

IP : 3.80 .181 .102

26 апреля 2023 г., 13:48:00

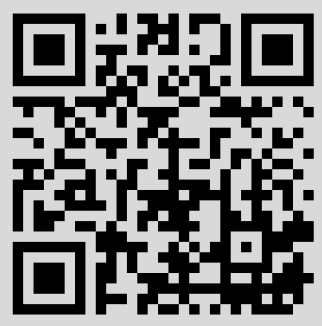


Вестн. Сам. гос. техн. ун-та. Сер. Физ.-мат. науки. 2016. Т. 20, № 4 . С. $739-754$

ISSN: 2310-7081 (online), 1991-8615 (print)

doi: http://dx.doi.org/10.14498/vsgtu1522

УДК 519.63:532.546

\title{
КВАЗИОДНОМЕРНАЯ МОДЕЛЬ ГИПЕРБОЛИЧЕСКОГО ТИПА ГИДРОРАЗРЫВА ПЛАСТА
}

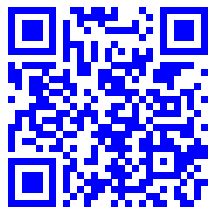

\section{А. М. Ильясов, Г. Т. Булгакова}

Уфимский государственный авиационный технический университет, Россия, 450000, Уфа, ул. К. Маркса, 12.

\begin{abstract}
Аннотация
Представлена квазиодномерная модель гиперболического типа развития трещины гидроразрыва пласта в предположении, что коэффициент интенсивности напряжений намного превосходит коэффициент трещиностойкости породы. Рассматриваемая модель, учитывающая конвективные и нестационарные слагаемые в уравнении движения жидкости, является обобщением локальной модели Перкинса-Керна-Нордгрена. Доказано, что полученная система дифференциальных уравнений является квазилинейной строго гиперболической системой, для которой найдены характеристики и соотношения на характеристиках. В случае пренебрежения поправкой Кориолиса найдены инварианты Римана. При пренебрежении фильтрационной утечкой и вязкостью закачиваемой жидкости определены волны Римана, аналогичные простым плоским волнам в газовой динамике, и изучены их свойства. Исследована эволюционность границ трещины гидроразрыва пласта. Поставлена начально-краевая задача развития трещины гидроразрыва пласта. Показано, что при пренебрежении диссипативными слагаемыми в представленной модели можно построить теорию простых волн, аналогичную теории одномерной газовой динамики с изоэнтропическими плоскими волнами.
\end{abstract}

Ключевые слова: гидроразрыв пласта, характеристики, инварианты Римана, эволюционность границ трещины.

Введение. В настоящей работе моделируется режим развития трещины гидроразрыва пласта (ГРП), в котором коэффициент интенсивности напряжений намного превосходит коэффициент трещиностойкости породы. В результате происходит разрушение породы на масштабах порядка размера пор в виде трещины-предвестника, фронт которой намного опережает фронт трещины ГРП, которая образуется вследствие нагнетания жидкости разрыва. В этом случае раскрытие вертикальной трещины ГРП происходит вследствие превышения давлением жидкости минимального горизонтального напряжения в породе и последующего заполнения уже существующей трещины-

(C) 2016 Самарский государственный технический университет.

\section{Образец для цитирования}

И ль я с о в А. М., Бу л г к о в а Г. Т. Квазиодномерная модель гиперболического типа гидроразрыва пласта // Вестн. Сам. гос. техн. ун-та. Сер. Физ.мат. науки, 2016. Т. 20, № 4. C. 739-754. doi: 10.14498/vsgtu1522.

\section{Сведения об авторах}

Айдар Мартисович Ильясов (к.ф.-м.н.; amilyasov67@gmail.com), старший научный сотрудник, каф. математики.

Гузель Талгатовна Булгакова (д.ф.-м.н.; bulgakova.guzel@mail.ru; автор, ведущий переписку), профессор, каф. математики. 
предвестника, образовавшейся в результате разрушения породы. Основы теории развития вертикальных трещин в пористой среде заложены в работе [1].

В последние два десятилетия как в отечественной, так и в зарубежной литературе появилось более двух десятков тысяч теоретических и прикладных работ по моделированию образования и развития трещин ГРП. В данном обзоре рассматриваются только квазиодномерные модели развития трещины ГРП, применение которых широко распространено в нефтедобывающей промышленности. В этих моделях жидкости гидроразрыва предполагаются ньютоновскими и несжимаемыми, а окружающий пористый массив считается линейно-упругим. Вследствие этого в одномерных моделях возникает линейная связь между давлением в трещине и ее раскрытием. По характеру этой связи различают локальные и нелокальные модели. В локальных моделях между раскрытием и давлением имеется конечная связь, а в нелокальных моделях эта связь представляет собой сингулярное интегральное уравнение [2]. Теоретические исследования этих моделей в основном посвящены нахождению автомодельных решений. В работах [3-6] изучаются локальные модели, а в работах [9-13] - нелокальные модели.

Модель распространения вертикальных трещин в пористой среде в случае ньютоновской жидкости гидроразрыва, когда длина трещины много больше ее постоянной высоты и ширины, разработана в работах [3,4] - локальная модель Перкинса-Керна-Нордгрена (модель PKN). В модели PKN течение в пласте не рассматривается и вводится эмпирический коэффициент фильтрационной утечки жидкости в пласт через стенки трещины. В рамках этой модели в работе [4] получены аналитические автомодельные решения в случаях малой и большой утечек, а также численные решения в общем случае.

В работе [5] рассмотрено обобщение классической модели PKN с зоной пропитки с прямолинейным фильтрационным течением, зависящим только от времени в несжимаемом пласте. Получены автомодельные решения в предельных случаях малых и больших фильтрационных утечек при заданном постоянном расходе или постоянном давлении на входе в трещину. В этих предельных случаях степенные зависимости искомых функций от времени близки к аналогичным зависимостям, полученным в [4]. Также в [5] показано, что в общем случае автомодельное решение возможно только при линейной зависимости давления от времени на входе в трещину.

В работе [7] предложена двухзональная модель PKN, которая обобщает модель, представленную в [5]. В работе [7] кроме зоны пропитки также учитывается изменение порового давления в дальней зоне пласта, где предполагается упругий режим фильтрации пластовой жидкости [6]. Методом последовательных приближений с применением многочленов Әрмита получено автомодельное решение при постоянном давлении на входе в трещину.

В работе [8] рассмотрена та же модель PKN с зоной пропитки, что и в [5]. Получены автомодельные решения степенного и экспоненциального вида в предельных случаях малых и больших фильтрационных утечек при расходе или давлении на входе в трещину такого же вида. В [8] показано, что в общем случае конечной фильтрационной утечки автомодельное степенное решение возможно только при линейной зависимости давления от времени на входе в трещину или квадратичной зависимости расхода от времени.

В работе [9] рассмотрена модель PKN с нелокальной связью раскрытия 
и давления в трещине. Получено автомодельное решение для раскрытия трещины и давления в виде разложения по полиномам Чебышева второго рода при постоянном расходе на входе и отсутствии фильтрационной утечки. Показано, что при умеренных расходах закачки форма трещины близка к эллиптической. При больших расходах градиент давления на конце трещины увеличивается, а длина трещины - уменьшается.

В рамках нелокальной модели с геометрией трещины, аналогичной геометрии классической модели $\mathrm{PKN}$, методами асимптотических разложений получены полуаналитические автомодельные решения для различных предельных режимов течения в трещине. В работе [10] получено полуаналитическое решение для режима течения в трещине с непроницаемыми стенками в пренебрежении коэффициентом трещиностойкости породы. В работе [11] получено решение для режима течения в трещине с непроницаемыми стенками с высоким коэффициентом трещиностойкости породы. В отличие от работ [10,11], в работе [12] представлен численный алгоритм для режима течения с фильтрационной утечкой, но с малой трещиностойкостью породы. Было проведено сравнение полученного численного решения на малых и больших временах с соответствующими асимптотическими решениями. Решение для наиболее общего случая, когда учитывается влияние всех трех механизмов на развитие трещины ГРП-коэффициента трещиностойкости, вязкости жидкости и фильтрационной утечки, получено в работе [13], в которой использован многопараметрический метод сингулярных возмущений. Для полученного решения рассмотрены предельные режимы малой и большой вязкости жидкости гидроразрыва. Во всех указанных работах используется приближение теории смазки [14]. Как следствие, локальные модели представляют собой системы уравнений параболического типа.

В данной работе рассматривается обобщение локальной модели PKN, учитывающее конвективные и нестационарные слагаемые в уравнении движения жидкости.

1. Физическое обоснование модели. В представленном выше обзоре рассмотрены только работы, в которых жидкости разрыва являются ньютоновскими. Это связано с тем, что во многих технологических процессах, связанных с ГРП, в качестве жидкости разрыва используется вода. К таким процессам относятся:

1) первая часть тестового (калибровочного) ГРП, в которой при скоростях закачки жидкости гидроразрыва пласта из скважины вытесняется пластовая вода линейным (несшитым) гелем; в этом случае в течение нескольких минут вытесняемой водой создается трещина ГРП;

2) ГРП в низкопроницаемых нефтенасыщенных пластах;

3) явление авто-ГРП - самопроизвольное распространение магистральной трещины от нагнетательной скважины.

Во всех перечисленных процессах вследствие малой вязкости воды в трещине ГРП могут существовать достаточно протяженные области, в которых силы инерции имеют один порядок с вязкими силами или превосходят их. Выполним оценку числа Рейнольдса Re. Предположим, что расход жидкости при ГРП равен $2 \div 4 \mathrm{~m}^{3} /$ мин. Тогда при высоте трещины $h$ порядка $10 \div 30 \mathrm{м}$ и ширине трещины $w$ у скважины (порядка диаметра перфорационных отверстий), равной 0.01 м, скорость течения в начале трещины $v$ будет составлять 
$0.11 \div 0.66 \mathrm{~m} /$ с. При этом $\mathrm{Re}=1100 \div 6600$. Если конечная длина трещины $L$ (полудлина, если трещина симметрична) равна $10 \div 50$ м, то соотношение инерционных и вязких сил для трещины в целом оценивается приведенным числом Рейнольдса $\operatorname{Re}_{L}=w \operatorname{Re} / L$, равным $0.2 \div 6.6$. Эта оценка показывает, что в случае, когда жидкостью разрыва является вода, нельзя пренебрегать силами инерции.

Характерное время $T$ тестового ГРП (наименее продолжительного из процессов 1-3) составляет 180 с. Если характерное расстояние $L_{0}$ от стенок трещины до границ пласта порядка 200 м, а скорость продольной волны в упругом пласте $a_{0}$ равна $4000 \mathrm{~m} / \mathrm{c}$, то характерное время пробега звуковой волны оценивается величиной $t_{0}=L_{0} / a_{0}$, равной 0.05 с. Отсюда следует оценка $T \gg t_{0}$. Следовательно, процесс упругого деформирования пласта при закачке жидкости в трещину можно считать квазистационарным.

\section{2. Квазиодномерная модель течения жидкости в вертикальной трещине.} Рассматривается процесс распространения вертикальной трещины постоянной высоты $h$ в насыщенном пористом пласте с коэффициентом проницаемости $k$ при нагнетании несжимаемой ньютоновской жидкости в пористый массив через вертикальную скважину. Предполагается, что трещина расположена симметрично относительно скважины.

Как и в модели PKN, предполагается, что высота трещины много больше ее ширины $\delta$ и много меньше ее длины $L$, т.е. выполняется неравенство $\delta \ll h \ll L$. Кроме этого, предполагается, что пласт является линейно-упругим, а по длине трещины условия напряженного состояния изменяются незначительно, что выполняется, если изменение давления жидкости внутри трещины много меньше характерного напряжения в пласте. Это дает возможность считать, что в плоскостях, перпендикулярных направлению трещины, приближенно выполняется условие плоской деформации, а сечения не влияют друг на друга (гипотеза плоских сечений $[3,4])$.

Принимается, что ось $0 x$ направлена вдоль трещины, ось $0 y$ направлена перпендикулярно оси $0 x$ в направлении изменения ширины трещины $\delta$, а ось $0 z$ направлена по высоте трещины. Далее считается, что трещина расположена симметрично относительно плоскости $x 0 y$, следовательно, верхний край трещины имеет координату $z=h / 2$, а нижний край - координату $z=-h / 2$.

В предыдущем пункте показано, что процесс упругого деформирования можно считать квазистационарным. Следовательно, можно использовать известное стационарное решение для ширины $\delta$ симметричной трещины, находящейся в условиях плоской деформации в полубесконечном пространстве, на контур которой действует постоянное давление [15]. При введенных выше допущениях для каждого поперечного сечения трещины ГРП можно приближенно написать следующее:

$$
\delta(x, z, t)=\frac{2(1-\nu)}{G}(p(x, t)-\sigma) \sqrt{\frac{h^{2}}{4}-z^{2}}
$$

где $\nu, G$ - соответственно коэффициент Пуассона и модуль сдвига породы; $p$ - давление в трещине; $\sigma$ - постоянное минимальное горизонтальное напряжение в пласте, в направлении которого трещина раскрывается при гидравлическом разрыве. 
Интегрирование (1) по высоте пласта позволяет найти площадь поперечного сечения как функцию координаты $x$ и времени $t$ :

$$
S(x, t)=\frac{\pi(1-\nu) h^{2}}{4 G}(p(x, t)-\sigma) .
$$

Если $S(x, t)$ - переменное поперечное сечение трещины, то с учетом несжимаемости жидкости можно ввести осредненные по сечению скорость, квадрат скорости и давление согласно выражениям

$$
\langle v\rangle=\frac{1}{S} \int_{S} v d s, \quad(1+\beta)\langle v\rangle^{2}=\frac{1}{S} \int_{S} v^{2} d s, \quad\langle p\rangle=\frac{1}{S} \int_{S} p d s .
$$

В подынтегральных выражениях в (3) записаны истинные скорости и давления, а $\beta$ - поправка Кориолиса на неравномерное распределение истинной скорости по сечению [16]. Для стационарных ламинарных течений с параболическим профилем скорости $\beta=1 / 3$, для квазистационарных турбулентных течений $\beta=0$. При неустановившихся течениях поправка Кориолиса является функцией времени.

С учетом (3) уравнения изменения массы и количества движения несжимаемой жидкости с постоянной плотностью $\rho$ в одномерном потоке в канале переменного сечения $S(x, t)$ с малой кривизной (необязательно вертикального) и проницаемыми стенками имеют вид

$$
\begin{gathered}
\frac{\partial(\rho S)}{\partial t}+\frac{\partial(\rho\langle v\rangle S)}{\partial x}=-\rho u_{L} \Pi \\
\frac{\partial(\rho\langle v\rangle S)}{\partial t}+\frac{\partial\left((1+\beta) \rho\langle v\rangle^{2} S\right)}{\partial x}=-S \frac{\partial\langle p\rangle}{\partial x}-\Pi \tau-\rho g S \sin \alpha,
\end{gathered}
$$

где $u_{L}$ - нормальная стенкам канала скорость фильтрационной утечки; П смоченный периметр; $\alpha$ - угол наклона канала к горизонту; $\tau$ - напряжение трения на стенке канала; $g$-ускорение силы тяжести. Как и в работе [5], можно заменить произвольное сечение $S(x, t)$ прямоугольным сечением с площадью, задаваемой соотношением (2):

$$
S(x, t)=w(x, t) h .
$$

Из (2) и (6) следует локальная связь между давлением в трещине и ее раскрытием $w$ - осредненной шириной трещины $\delta$ по ее высоте $h$ :

$$
p-\sigma=b w, \quad b=\frac{4 G}{\pi h(1-\nu)}>0
$$

где $b$ - жесткость трещины.

С учетом (7) система уравнений (4), (5) запишется в дивергентном виде:

$$
\begin{gathered}
\frac{\partial(w)}{\partial t}+\frac{\partial(w v)}{\partial x}=-2 u_{L} \\
\frac{\partial(w v)}{\partial t}+\frac{\partial\left((1+\beta) w v^{2}+b w^{2} /(2 \rho)\right)}{\partial x}=-2 \frac{\tau}{\rho}
\end{gathered}
$$


где учтено, что трещина вертикальна, а смоченный периметр, в силу допущений модели, следующий:

$$
\Pi(x, t)=2 w(x, t)+2 h \approx 2 h .
$$

Скобки осреднения в уравнениях опущены.

Систему (8), (9) также можно представить в неконсервативном виде:

$$
\begin{aligned}
& A \frac{\partial \boldsymbol{U}}{\partial t}+B \frac{\partial \boldsymbol{U}}{\partial x}=\boldsymbol{d}, \quad \boldsymbol{U}=(w, v)^{\top}, \quad A=\left(\begin{array}{cc}
1 & 0 \\
v & w
\end{array}\right) \\
& \left(\begin{array}{cc}
v & w \\
(1+\beta) v^{2}+b w / \rho & 2(1+\beta) w v
\end{array}\right)=B, \quad \boldsymbol{d}=\left(-2 u_{L},-2 \tau / \rho\right)^{\top} .
\end{aligned}
$$

Отсюда следует, что данная система уравнений является квазилинейной.

Для замыкания системы уравнений (8), (9) или (10), (11) необходимо задать скорость фильтрации жидкости через ее стенки и напряжение трения на стенках трещины.

Далее предполагается следующее:

1) пласт бесконечен и в начальный момент времени пластовое (поровое) давление постоянно и равно $p_{k}$;

2) течение в пласте квазистационарно и подчиняется уравнениям упругого режима фильтрации;

3) в каждом сечении трещины фильтрационная утечка жидкости через стенки трещины не зависит от утечек в соседних сечениях и представляет собой прямолинейно-параллельный поток в пласте.

При допущениях 1-3 применение автомодельного решения для прямолинейного течения в бесконечном линейно-упругом пласте при заданном постоянном давлении на границе полупространства (течение от нагнетательной галереи) [6] приводит к двухмерному распределению давления $p(x, y, t)$ в пласте:

$$
p(x, y, t)=p(x, t)+\left(p_{k}-p(x, t)\right) \operatorname{erf}\left(\frac{y}{2 \sqrt{\chi t}}\right),
$$

где $\chi=k /\left(\mu \beta_{*}\right)$ - коэффициент пъезопроводности породы; $\beta_{*}=\beta_{l} m+\beta_{s}-$ коэффициент упругой сжимаемости пласта; $\beta_{l} m, \beta_{s}-$ соответственно коэффициенты упругой сжимаемости пластовой жидкости и скелета породы.

С учетом (12) скорость фильтрационной утечки жидкости через стенки трещины принимается в виде

$$
u_{L}=-\left.\frac{k}{\mu} \frac{\partial p(x, y, t)}{\partial y}\right|_{y=0}=\frac{k}{\mu} \frac{\left(p(x, t)-p_{k}\right)}{\sqrt{\pi \chi\left(t-t_{L}\right)}}
$$

где $k$ - проницаемость пласта; $\mu$ - вязкость закачиваемой жидкости. В формуле (13) учтено запаздывание утечки вследствие распространения фронта трещины. В формуле (13) обозначено $t_{L}=f^{-1}(L)$, а $L=f(t)$ - закон движения фронта трещины.

Нужно отметить, что допущения 1-3 являются модельными и вместо (13) можно использовать другие эмпирические или аналитические выражения для 
скорости фильтрационной утечки. При известной поправке Кориолиса $\beta$ полученная система уравнений (8), (9) замыкается заданием напряжения трения $\tau$ и коэффициента трения $C_{w}$ при ламинарном режиме $(\operatorname{Re} \leqslant 2000)$ течения [17]:

$$
\tau=C_{w} \frac{\rho v^{2}}{2}, \quad C_{w}=\frac{\lambda}{4}=\frac{k_{0} \cdot 64}{4 \mathrm{Re}}=\frac{24}{\mathrm{Re}},
$$

где $k_{0}=3 / 2$ - поправочный коэффициент для каналов прямоугольного сечения, когда одна сторона прямоугольника много больше другой его стороны; $\lambda$ - коэффициент гидравлического сопротивления. В формуле (14) число Рейнольдса $\operatorname{Re}$ определяется через гидравлический диаметр $D_{H}$ сечения трещины:

$$
\operatorname{Re}=\frac{\rho v D_{H}}{\mu}, \quad D_{H}=\frac{4 S}{\Pi}=\frac{4 w h}{2(w+h)} \approx 2 w .
$$

При турбулентном режиме $(\operatorname{Re}>2000)$ течения [17]:

$$
\tau=C_{w} \frac{\rho v^{2}}{2}, \quad C_{w}=\frac{\lambda_{1}}{4}=\frac{\lambda k_{0}}{4}=\frac{k_{0} \cdot 0.3164}{4 \operatorname{Re}^{0.25}}=\frac{0.08701}{\operatorname{Re}^{0.25}},
$$

где пренебрегаем шероховатостью стенок трещины. Здесь $\lambda_{1}-$ коэффициент гидравлического сопротивления при турбулентном режиме течения в канале прямоугольного сечения; $\lambda$ - коэффициент гидравлического сопротивления при турбулентном режиме течения в круглой трубе (формула Блазиуса); $k_{0}=1.1$ - поправочный коэффициент для турбулентных потоков в каналах прямоугольного сечения, когда одна сторона прямоугольника много больше другой его стороны.

3. Характеристики, соотношения на характеристиках и инварианты Римана. Матрица $A$ в $(10)$ не вырождена при условии $w \neq 0$. Умножение векторного уравнения (10) слева на обратную матрицу $A^{-1}$ дает новое представление данного уравнения:

$$
\begin{gathered}
\frac{\partial \boldsymbol{U}}{\partial t}+C \frac{\partial \boldsymbol{U}}{\partial x}=\boldsymbol{f}, \quad C=A^{-1} B=\left(\begin{array}{cc}
v & w \\
\left(\beta v^{2}+\rho^{-1} b w\right) / w & (2 \beta+1) v
\end{array}\right), \\
\boldsymbol{f}=A^{-1} \boldsymbol{d}=\left(-2 u_{L}, 2\left(u_{L} v-\tau / \rho\right) / w\right)^{\top}, \quad \boldsymbol{U}=(w, v)^{\top} .
\end{gathered}
$$

Согласно общей теории квазилинейных уравнений [18-21], вычисляются собственные значения матрицы $C$, что при условии $w \neq 0$ дает два вещественных собственных значения на решении $\boldsymbol{U}$ :

$$
\lambda_{ \pm}=(\beta+1) v \pm \sqrt{\left(\beta^{2}+\beta\right) v^{2}+b w / \rho} .
$$

Дифференциальные уравнения характеристических кривых имеют следующий вид:

$$
\begin{array}{ll}
C_{+}: & \frac{d x}{d t}=(\beta+1) v+\sqrt{\left(\beta^{2}+\beta\right) v^{2}+b w / \rho} \\
C_{-}: & \frac{d x}{d t}=(\beta+1) v-\sqrt{\left(\beta^{2}+\beta\right) v^{2}+b w / \rho}
\end{array}
$$


Находятся левые собственные векторы:

$$
\begin{aligned}
& \boldsymbol{l}_{\mathbf{1}}=\left(\frac{1}{w}\left(-\beta v+\sqrt{\left(\beta^{2}+\beta\right) v^{2}+b w / \rho}\right), 1\right)^{\top} \\
& \boldsymbol{l}_{\mathbf{2}}=\left(\frac{1}{w}\left(-\beta v-\sqrt{\left(\beta^{2}+\beta\right) v^{2}+b w / \rho}\right), 1\right)^{\top} .
\end{aligned}
$$

Матрица $\Omega_{L}$, составленная из левых собственных векторов, не вырождена при условии $w \neq 0$, поскольку

$$
\operatorname{det} \Omega_{L}=\operatorname{det}\left(\begin{array}{ll}
l_{11} & l_{12} \\
l_{21} & l_{22}
\end{array}\right)=\frac{2}{w} \sqrt{\left(\beta^{2}+\beta\right) v^{2}+b w / \rho} \neq 0,
$$

где номер строки обозначает номер собственного вектора, а номер столбца его координату. Таким образом, система уравнений $(16),(17)$ является строго гиперболической системой [18].

Соотношения на характеристиках имеют следующий вид:

$$
\begin{aligned}
& \frac{1}{w}\left(-\beta v+\sqrt{\left(\beta^{2}+\beta\right) v^{2}+b w / \rho}\right) D_{+} w+D_{+} v= \\
& =-\frac{2}{w}\left(-\beta v+\sqrt{\left(\beta^{2}+\beta\right) v^{2}+b w / \rho}\right) u_{L}+\frac{2}{w}\left(u_{L} v-\tau / \rho\right)
\end{aligned}
$$

$$
\begin{aligned}
& \frac{1}{w}\left(-\beta v-\sqrt{\left(\beta^{2}+\beta\right) v^{2}+b w / \rho}\right) D_{-} w+D_{-} v= \\
& =\frac{2}{w}\left(\beta v+\sqrt{\left(\beta^{2}+\beta\right) v^{2}+b w / \rho}\right) u_{L}+\frac{2}{w}\left(u_{L} v-\tau / \rho\right) .
\end{aligned}
$$

Здесь введены операторы внутреннего дифференцирования вдоль характеристик [20]:

$$
D_{+}=\partial_{t}+\lambda_{+} \partial_{x}, \quad D_{-}=\partial_{t}+\lambda_{-} \partial_{x},
$$

а в правых частях (21) и (22) скорость фильтрации в пласт и пристеночное трение выражаются через искомые функции по формулам (13)-(15).

Как известно [19], квазилинейную гиперболическую систему из двух уравнений можно записать в инвариантах Римана. Однако при любом $\beta>0$ инварианты Римана системы (21), (22) не выражаются в элементарных функциях. Это удается сделать, если пренебречь поправкой Кориолиса, т.е. положить $\beta=0$. В этом случае соотношения вдоль характеристик запишутся в виде

$$
\begin{gathered}
D_{+} I_{1}=-\frac{2}{w} \sqrt{b w / \rho} u_{L}+\frac{2}{w}\left(u_{L} v-\tau / \rho\right), \\
D_{-} I_{2}=\frac{2}{w} \sqrt{b w / \rho} u_{L}+\frac{2}{w}\left(u_{L} v-\tau / \rho\right),
\end{gathered}
$$

где инварианты Римана суть

$$
I_{1,2}=v \pm 2 \sqrt{b w / \rho}
$$


Правые части соотношений (23) и (24) выражаются через инварианты Римана с учетом (7), (13)-(15) и следующих из (25) представлений искомых функций через эти инварианты:

$$
w=\frac{\rho}{16 b}\left(I_{1}-I_{2}\right)^{2}, \quad v=\frac{1}{2}\left(I_{1}+I_{2}\right) .
$$

При $\beta=0$ дифференциальные уравнения характеристик (18), (19) имеют вид

а величину

$$
C_{+}: \frac{d x}{d t}=v+\sqrt{b w / \rho}, \quad C_{-}: \frac{d x}{d t}=v-\sqrt{b w / \rho},
$$

$$
a=\sqrt{b w / \rho}=\sqrt{(p-\sigma) / \rho}
$$

можно трактовать как скорость малых возмущений раскрытия трещины или давления (играет роль скорости звука). Отметим, что (27) совпадает со скоростью малых возмущений давления в трубке с упругими стенками, вычисленной для линейной связи (7) через растяжимость стенок трубки [22].

Для наглядности на рисунке показаны зависимости местной скорости $a$ малых возмущений давления (27) несжимаемой жидкости с плотностью $\rho=$ $=1000 \mathrm{kг} / \mathrm{M}^{3}$ от раскрытия $w$ трещины для различных значений высоты трещины $h$ для породы с модулем сдвига $G=25$ ГПа и коэффициентом Пуассона $\nu=0.13$ (песчаник).

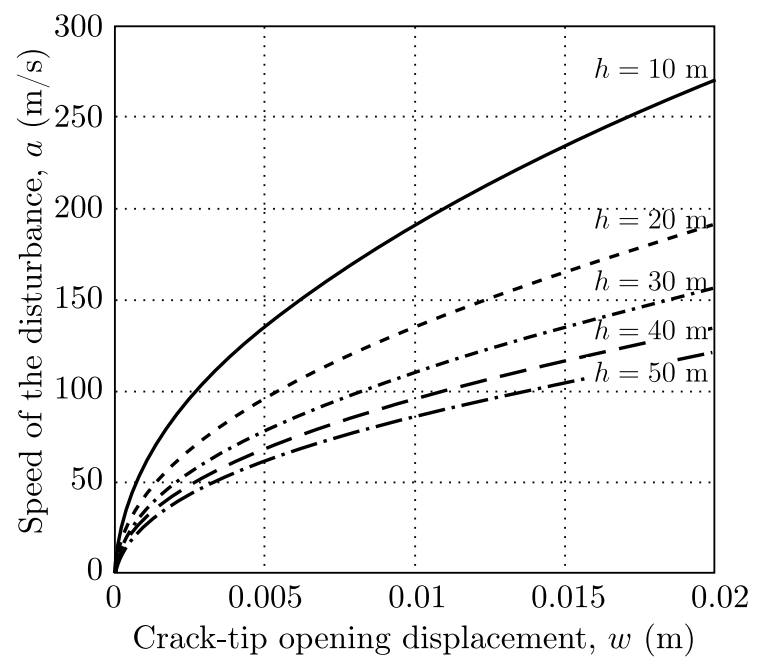

Зависимость местной скорости малых возмущений давления $a$ от раскрытия трещины $w$ для различных значений высоты трещины $h$ [The dependence of local speed of the pressure disturbance on the crack-tip opening displacement for different values of fracture height]

Очевидно, что уравнения модели развития трещины гидроразрыва и все предыдущие соотношения можно легко переписать в терминах скорости и давления жидкости.

4. Граничные условия для трещины ГРП. Эволюционность границ трещины. Постановка начально-краевой задачи. Далее рассматриваются вопросы о граничных и начальных условиях уравнений представленной модели развития трещины гидроразрыва пласта. Вопросы корректности начально-краевых 
задач для систем квазилинейных гиперболических уравнений и уравнений газовой динамики рассмотрены в [19]. Вопросы о количестве граничных условий на подвижных и неподвижных границах для уравнений газовой динамики рассмотрены в [19,21,23], а для квазилинейных уравнений - в [18, 19,23].

Трещина ГРП образуется в области перфорационных отверстий вертикальной скважины, т.е. входная граница является неподвижной. Обычно на практике при инициации трещины ГРП задается постоянный расход $Q_{0}$ закачиваемой жидкости, поэтому граничным условием на входе трещины ГРП является следующее нелинейное граничное условие:

$$
Q_{0}-h w v=0 .
$$

Рассмотрим вопрос об эволюционности входной границы трещины ГРП. Пусть в области $x>0$ в начальный момент времени заданы начальные условия. Из области течения $x>0$ к неподвижной границе $x=0$ приходит одна характеристика с характеристической скоростью $\lambda_{-}=v-a<0$. От входной границы в область течения уходит характеристика второго семейства $\lambda_{+}=$ $=v+a>0$. Входная граница будет эволюционной, если на ней задано одно граничное условие (28), так как в этом случае на границе определяются раскрытие $w$ и скорость течения $v$.

Предположим, что фронт трещины представляет собой сильный разрыв ее раскрытия (давления). В случае пренебрежения неоднородностью профиля скорости $(\beta=0)$ применение интегральных законов сохранения массы и количества движения [21] к установившемуся квазиодномерному течению в канале с упругими стенками с учетом сил давления, возникающих на скачке в системе координат, связанной с разрывом, приводит к следующим условиям на скачке:

$$
\begin{gathered}
S_{2} v_{2}=S_{1} v_{1}-\int_{1}^{2} \Pi u_{L} d x \\
S_{2}\left(p+\rho v_{2}^{2}\right)=S_{1}\left(p+\rho v_{1}^{2}\right)+\int_{1}^{2} p(S) d S-\int_{1}^{2} \Pi \tau d x-\rho \int_{1}^{2} \Pi v u_{L} d x .
\end{gathered}
$$

Здесь считается, что скачок раскрытия имеет некоторую ширину. Индексом 1 обозначены параметры перед разрывом, а индексом 2 - параметры за разрывом. Для простоты предполагается, что плотности закачиваемой и пластовой жидкостей совпадают.

При пренебрежении фильтрационной утечкой и вязкостью жидкости эти соотношения совпадают с условиями на ударной волне в идеальной несжимаемой жидкости [22] в трубке с упругими стенками, если связь $p=p(S)$ имеет место на ударной волне.

Поскольку на практике выполняется неравенство $u_{L} \ll v$, последними интегралами в правых частях законов сохранения можно пренебречь. Если, кроме того, второй интеграл в правой части закона сохранения количества движения много меньше первого, то в системе координат наблюдателя в случае линейной связи (7) условия на скачке раскрытия примут вид

$$
\begin{gathered}
w_{1}\left(v_{1}-D\right)=w_{2}\left(v_{2}-D\right)=m, \\
\rho w_{1}\left(v_{1}-D\right)^{2}+\frac{b}{2} w_{1}^{2}=\rho w_{2}\left(v_{2}-D\right)^{2}+\frac{b}{2} w_{2}^{2} .
\end{gathered}
$$


В формулах (29), (30) $D$ - скорость скачка; $m$ - поток массы нагнетаемой жидкости через разрыв.

Далее рассматривается вопрос об эволюционности фронта трещины ГРП, который, по предположению, является сильным разрывом раскрытия трещины. В этом случае $m \neq 0$ и жидкость течет через разрыв. Рассмотрим течение жидкости разрыва в области $x \geqslant 0$ с подвижной внутренней границей $x=x(t)$ - сильным скачком раскрытия трещины ГРП, если заданы начальные условия и краевое условие (28). На скачке заданы пять неизвестных величин $w_{1}, v_{1}, w_{2}, v_{2}, D$. Для того чтобы разрыв раскрытия трещины был эволюционным, число заданных граничных условий на нем должно составлять $N=5-s$, где $s$ - общее число приходящих на разрыв характеристик из областей течения слева и справа от разрыва.

В [22] показано, что в ударных волнах в упругой трубке, аналогично ударным волнам в газовой динамике, скорость течения жидкости относительно скачка перед скачком сверхкритическая $\left(\left|v_{1}-D\right|>a_{1}\right)$, а за скачком - докритическая $\left(\left|v_{2}-D\right|<a_{2}\right)$. Это означает, что из области слева от разрыва на скачок приходит одна характеристика со скоростью $\lambda_{+}=v_{2}-D+a>0$, а из области справа от разрыва на скачок приходят две характеристики со скоростями $\lambda_{+}=v_{1}-D+a<0$ и $\lambda_{-}=v_{1}-D-a<0$. Итак, $s=3, N=2$, и задания двух законов сохранения (29) и (30) на скачке достаточно для его эволюционности.

В случае $m=0$ жидкость не течет через разрыв. Из (29) и (30) следует, что в этом случае течение на фронте трещины ГРП является непрерывным, поскольку выполняются равенства $w_{1}=w_{2}, v_{1}=v_{2}$. Скорость скачка в этом случае является скоростью распространения фронта трещины.

Поскольку в данной модели развитие трещины разрыва породы не моделируется, предлагается следующая идеализированная постановка задачи о развитии трещины ГРП: при $t>0$ в области $x \geqslant 0$ найти обобщенное решение [19] гиперболической системы уравнений (16), (17) с граничным условием (28) на входной границе, условиями на бесконечности

$$
\lim _{x \rightarrow \infty} w(x, t)=w_{0} \neq 0, \quad \lim _{x \rightarrow \infty} v(x, t)=0
$$

и начальными условиями

$$
w(x, 0)=w_{0} \neq 0, \quad v(x, 0)=0 .
$$

Требование неравенства нулю раскрытия трещины в начальный момент времени следует из условия (20) гиперболичности системы уравнений (16), (17).

5. Аналогия с газовой динамикой. Для численного решения полученной системы уравнений методами типа Годунова [18] необходимо решить задачу о распаде произвольного начального разрыва в пренебрежении диссипативными слагаемыми - трением в трещине ГРП и фильтрационной утечкой. В этом случае при $\beta=0$ система уравнений (16), (17) с учетом (27) примет вид

$$
\begin{aligned}
& \frac{\partial w}{\partial t}+v \frac{\partial w}{\partial x}+w \frac{\partial v}{\partial x}=0, \\
& \frac{\partial v}{\partial t}+\frac{a^{2}}{w} \frac{\partial w}{\partial x}+v \frac{\partial v}{\partial x}=0 .
\end{aligned}
$$


Уравнения (31) и (32) аналогичны уравнениям газовой динамики в гидравлическом приближении [23], если в последних уравнениях газ считать несжимаемой жидкостью. Можно также заметить, что существует аналогия с уравнениями одномерной газовой динамики с изоэнтропическими плоскими волнами [19-21], если учесть, что в уравнениях (31) и (32) раскрытие трещины играет одновременно как роль плотности, так и роль давления. Применительно к системе уравнений (31), (32) можно ввести определения в терминах раскрытия трещины $w$ и скорости жидкости $v$, инварианты Римана $I_{1}, I_{2}[22]$, а также получить решения типа простых волн (ПВ): волн Римана (BР) и центрированных волн и доказать утверждения, аналогичные утверждениям в одномерной газовой динамике с изоэнтропическими плоскими волнами [20].

1. Вырожденная ПВ системы (31), (32) есть постоянное течение. Ее линиями уровня являются траектории частиц жидкости, покрывающие плоскость событий. Линиями уровня невырожденной ПВ являются прямолинейные характеристики (26), покрывающие плоскость событий.

2. Течение, удовлетворяющее системе уравнений (31), (32), является невырожденной ПВ тогда и только тогда, когда один из инвариантов Римана тождественно постоянен. Если $I_{1} \equiv$ const, то линии уровня ПВ - прямолинейные характеристики $C_{-}$. Если $I_{2} \equiv$ const, то линиями уровня ПВ являются прямолинейные характеристики $C_{+}$.

3. (Достаточный признак ПВ) Если в непрерывном течении имеется характеристика $C_{+}\left(C_{-}\right)$, вдоль которой постоянны $w$ и $v$, причем $w \neq 0$, то с каждой ее стороны течение является либо постоянным, либо ВР.

4. Невырожденная ВР является волной сжатия (разрежения) тогда и только тогда, когда ее прямолинейные характеристики с ростом времени сходятся (расходятся) на плоскости событий. В волне сжатия частицы разгоняются, а в волне разрежения - тормозятся.

Заключение. В работе предложена математическая модель развития трещины ГРП гиперболического типа, являющаяся обобщением модели PKN. Исследована эволюционность границ трещины ГРП. Предложена постановка начально-краевой задачи для представленных уравнений модели. Показано, что при пренебрежении диссипативными слагаемыми в представленной модели можно построить теорию простых волн, аналогичную теории одномерной газовой динамики с изоэнтропическими плоскими волнами.

Декларация о финансовых и других взаимоотношениях. Исследование выполнено при финансовой поддержке Минобрнауки РФ в рамках базовой части государственного задания организациям высшего образования в 2016 г. и РФФИ (проект № 14-01-97012 p). Все авторы принимали участие в разработке концепции статьи и в написании рукописи. Авторы несут полную ответственность за предоставление окончательной рукописи в печать. Окончательная версия рукописи была одобрена всеми авторами. Авторы не получали гонорар за статью.

\section{ORCID}

Айдар Мартисович Ильясов: http://orcid.org/0000-0002-4399-9040

Гузель Талгатовна Булгакова: http://orcid.org/0000-0001-8030-1791

\section{БИБЛИОГРАФИЧЕСКИЙ СПИСОК}

1. Желтов Ю. П., Христианович С. А. О гидравлическом разрыве нефтеносного пласта // Изв. АН СССР. ОТН, 1955. № 5. С. 3-41. 
2. Мусхелишвили Н. Н. Некоторые основные задачи математической теории упругости. М.: Наука, 1966. 707 с.

3. Perkins T. K., Kern. L. R. Widths of Hydraulic Fractures // J. Petroleum Technol., 1961. vol. 13, no. 9. pp. 937-949. doi: 10.2118/89-pa.

4. Nordgren R. P. Propogation of a vertical hydraulic fracture // Society of Petroleum Engineers Journal, 1972. vol. 12, no. 4. pp. 306-314. doi: 10.2118/3009-pa.

5. Ивашнев О. Е., Смирнов Н. Н. Формирование трещины гидроразрыва в пористой среде// Вестн. МГУ. Сер. 1. Математика, Механика, 2003. №6. С. 28-37.

6. Басниев К. С., Кочина И. Н., Максимов В. М. Подземная гидромеханика. М.: Недра, 1993. $416 \mathrm{c}$.

7. Гордеев Ю. Н., Зазовский А. Ф. Автомодельное решение задачи о глубокопроникающем гидравлическом разрыве пласта // Изв. РАН. МTT, 1991. № 5. С. 119-131.

8. Смирнов Н. Н., Тагирова В. Р. Автомодельные решения задачи о формировании трещины гидроразрыва в пористой среде // Изв. РАН. МЖК, 2007. № 1. С. 70-82.

9. Теодорович Э. В., Трофимов А. А., Шумилин И. Д. Форма плоской трещины гидроразрыва в упругой непроницаемой среде при различных скоростях закачки // Изв. PAН. МЖГ, 2011. № 4. С. 109-118.

10. Garagash D. I., Detournay E. Plane-strain propagation of a hydraulic fracture: small toughness solution // J. Appl. Mech., 2005. vol. 72, no.6. pp. 916-928. doi: 10.1115/1.2047596.

11. Garagash D. I. Plane strain propagation of a hydraulic fracture during injection and shut-in: large toughness solutions // Engng. Fract. Mech., 2006. vol. 73, no. 4. pp. 456-481. doi: 10. 1016/j.engfracmech.2005.07.012.

12. Adachi J. I., Detournay E. Plane-strain propagation of a hydraulic fracture in a permeable rock// Engng. Fract. Mech., 2008. vol.75, no.16. pp. 4666-4694. doi:10.1016/j. engfracmech.2008.04.006.

13. Mitchell S. L., Kuske R., Peirce A. P. An asymptotic framework for finite hydraulic fractures including leak-off// SIAM J. Appl. Math., 2007. vol.67, no.2. pp. 346-386. doi: 10.1137/ 04062059x.

14. Лойцянский Л. Г. Механика жидкости и газа. М.: Наука, 1987. 840 с.

15. Sneddon I. N., Berry D. S. The classical theory of elasticity / Elasticity and Plasticity / Encyclopedia of Physics, 6; ed. S. Flügge. Berlin: Springer-Verlag, 1958. pp. 1-126. doi : 10. 1007/978-3-662-43081-1_1.

16. Чарный И. А. Неустановившееся движение реальной жидкости в трубах. М.: Недра, 1975. 296 c.

17. Идельчик И. Е. Справочник по гидравлическим сопротивлениям. М.: Машиностроение, 1992. $672 \mathrm{c.}$

18. Куликовский А. Г., Погорелов Н. В., Семенов А. Ю. Математические вопросы численного решения гиперболических систем уравнений. М.: Физматлит, 2001. 608 с.

19. Рождественский Б. Л., Яненко Н. Н. Системы квазилинейных уравнений и их приложения к газовой динамике. М.: Наука, 1978. 688 с.

20. Овсянников Л. В. Лекции по основам газовой динамики. М., Ижевск: Инс-т комп. иссл., 2003. 336 c.

21. Черный Г. Г. Газовая динамика. М.: Наука, 1988. 424 с.

22. Pedley T. J. The Fluid Mechanics of Large Blood Vessels / Cambridge Monographs on Mechanics and Applied Mathematics. Cambridge: Cambridge University Press, 1980. xv+446 pp. doi: $10.1017 /$ cbo9780511896996.

23. Зверев И. Н., Смирнов Н. Н. Газодинамика горения. М.: МГУ, 1987. 307 с.

Поступила в редакцию $17 / \mathrm{XI} / 2016$;

в окончательном варианте - 05/XII/2016;

принята в печать - 09/XII/2016. 
Vestn. Samar. Gos. Techn. Un-ta. Ser. Fiz.-mat. nauki

[J. Samara State Tech. Univ., Ser. Phys. \& Math. Sci.], 2016, vol. 20, no. 4, pp. 739-754

ISSN: 2310-7081 (online), 1991-8615 (print)

doi: http://dx.doi.org/10.14498/vsgtu1522

MSC: 74F10, 74R10, 76D99

\title{
THE QUASI-ONE-DIMENSIONAL HYPERBOLIC MODEL OF HYDRAULIC FRACTURING
}

\author{
A. M. Ilyasov, G. T. Bulgakova \\ Ufa State Aviation Technical University, \\ 12, K. Marx st., Ufa, 450000, Russian Federation.
}

\begin{abstract}
The paper describes a quasi-one-dimensional hyperbolic model of hydraulic fracture growth assuming for the hydraulic fracturing that stress intensity is much higher than fracture resistance. The mode under analysis, which accounts for convective and unsteady terms in the fluid flow equation, is a generalization of the Perkins-Kern-Nordgren local model. It has been proved that the obtained system of differential equations is a quasi-linear strictly hyperbolic system, for which the characteristics were found as well as their correlations. For the case of the Coriolis correction neglect, the Riemann invariants were found. Neglecting the injected fluid leak-off and viscosity, the Riemann waves, similar to simple plane waves in gas dynamics, were defined and their properties were studied. The evolutionism of fracture boundaries was investigated. The initial boundary value problem was set for fracture growth. It has been shown that the neglect of dissipative terms in the presented model allows constructing a simple wave theory analogous to the theory of one-dimensional gas dynamics for isentropic plane waves.
\end{abstract}

Keywords: hydraulic fracturing, characteristics, Riemann invariants, fracture evolution.

Declaration of Financial and Other Relationships. This work was supported by the Ministry of Education and Science of the Russian Federation in the framework of the basic tasks of the state educational institutions of higher education in 2016 and supported by the Russian Foundation for Basic Research (project no. 14-01-97012 r povolzh'e_a). Each author has participated in the article concept development and in the manuscript writing. The authors are absolutely responsible for submitting the final manuscript in print. Each author has approved the final version of manuscript. The authors have not received any fee for the article.

(C) 2016 Samara State Technical University.

Please cite this article in press as:

Ilyas ov A. M., B ulgakova G. T. The quasi-one-dimensional hyperbolic model of hydraulic fracturing, Vestn. Samar. Gos. Tekhn. Univ., Ser. Fiz.-Mat. Nauki [J. Samara State Tech. Univ., Ser. Phys. \& Math. Sci.], 2016, vol. 20, no. 4, pp. 739-754. doi: 10.14498/vsgtu1522. (In Russian)

\section{Authors Details:}

Aidar M. Ilyasov (Cand. Phys. \& Math. Sci.; amilyasov67@gmail.com), Senior Researcher, Dept. of Mathematics.

Guzel T. Bulgakova (Dr. Phys. \& Math. Sci.; bulgakova.guzel@mail.ru; Corresponding Author), Professor, Dept. of Mathematics. 


\author{
ORCIDs \\ Aidar M. Ilyasov: http://orcid.org/0000-0002-4399-9040 \\ Guzel T. Bulgakova: http://orcid.org/0000-0001-8030-1791
}

\title{
REFERENCES
}

1. Zheltov Yu. P., Khristianovich S. A. On Hydraulic Fracturing of Oil Reservoirs, Izv. Akad. Nauk SSSR, Otd. Tekh. Nauk, 1955, no. 5, pp. 3-41 (In Russian).

2. Muskhelishvili N. I. Some Basic Problems of the Mathematical Theory of Elasticity. Netherlands, Springer, 1977, xxxi+732 pp. doi : 10.1007/978-94-017-3034-1.

3. Perkins T. K., Kern. L. R. Widths of Hydraulic Fractures, J. Petroleum Technol., 1961, vol. 13, no. 9, pp. 937-949. doi: 10.2118/89-pa.

4. Nordgren R. P. Propogation of a vertical hydraulic fracture, Society of Petroleum Engineers Journal, 1972, vol. 12, no. 4, pp. 306-314. doi: 10.2118/3009-pa.

5. Ivashnev O. E., Smirnov N. N. Formation of a Hydraulic Fracture in a Porous Medium, Vestn. MGU. Matematika, Mekhanika, 2003, no. 6, pp. 28-37 (In Russian).

6. Basniev K. S., Kochina I. N., Maksimov V. M. Podzemnaia gidromekhanika [Underground fluid mechanics]. Moscow, Nedra, 1993, 416 pp. (In Russian)

7. Gordeyev Yu. N., Zazovsky A. F. Self-similar solution for deep-penetrating hydraulic fracture propagation, Transp. Porous. Med., 1992, vol.7, no.3, pp. 283-304. doi:10.1007/ BF01063964.

8. Smirnov N. N., Tagirova V. P. Self-similar solutions of the problem of formation of a hydraulic fracture in a porous medium, Fluid Dynamics, 2007, vol.42, no. 1, pp. 60-70. doi : 10.1134/S0015462807010073.

9. Teodorovich E. V., Trofimov A. A., Shumilin I. D. Shape of a plane hydraulic fracture crack in an elastic impermeable medium at various injection rates, Fluid Dynamics, 2011, vol. 46, no. 4, pp. 603-612. doi: 10.1134/S0015462811040107.

10. Garagash D. I., Detournay E. Plane-strain propagation of a hydraulic fracture: small toughness solution, J. Appl. Mech., 2005, vol.72, no.6, pp. 916-928. doi: 10.1115/1.2047596.

11. Garagash D. I. Plane strain propagation of a hydraulic fracture during injection and shut-in: large toughness solutions, Engng. Fract. Mech., 2006, vol.73, no.4, pp. 456-481. doi: 10. 1016/j . engfracmech.2005.07.012.

12. Adachi J. I., Detournay E. Plane-strain propagation of a hydraulic fracture in a permeable rock, Engng. Fract. Mech., 2008, vol.75, no. 16, pp. 4666-4694. doi: 10.1016/j. engfracmech.2008.04.006.

13. Mitchell S. L., Kuske R., Peirce A. P. An asymptotic framework for finite hydraulic fractures including leak-off, SIAM J. Appl. Math., 2007, vol.67, no.2, pp. 346-386. doi: 10.1137/ 04062059x.

14. Loitsiansky L. G. Mekhanika zhidkosti i gaza [Mechanics of liquid and gas]. Moscow, Nauka, 1987, 840 pp. (In Russian)

15. Sneddon I. N., Berry D. S. The classical theory of elasticity, In: Elasticity and Plasticity, Encyclopedia of Physics, 6; ed. S. Flügge. Berlin, Springer-Verlag, 1958, pp. 1-126. doi: 10. 1007/978-3-662-43081-1_1.

16. Charnyi I. A. Neustanovivsheesia dvizhenie real'noi zhidkosti v trubakh [Unsteady motion of a real fluid in pipes]. Moscow, Nedra, 1975, 296 pp. (In Russian)

17. Idelchik I. E. Handbook of Hydraulic Resistance. Jerusalem, Israel Program for Scientific Translations, 1966, viii +517 pp.

18. Kulikovskii A. G., Pogorelov N. V., Semenov A. Yu. Mathematical aspects of numerical solution of hyperbolic systems, Monographs and Surveys in Pure and Applied Mathematics, vol. 118. Boca Raton, FL, Chapman \& Hall/CRC, 2001, xiii +540 pp.

19. Rozhdestvenskij B. L., Yanenko N. N. Systems of quasilinear equations and their applications to gas dynamics, Translations of Mathematical Monographs, vol. 55. Providence, R.I., AMS, 1983, $\mathrm{xx}+676 \mathrm{pp}$. 
20. Ovsyannikov L. V. Lektsii po osnovam gazovoi dinamiki [Lectures on the Fundamentals of Gas Dynamics]. Moscow, Izhevsk, Institute of computer investigations, 2003, 336 pp. (In Russian)

21. Chernyi G. G. Gazovaia dinamika [Gas Dynamics]. Moscow, Nauka, 1988, 424 pp. (In Russian)

22. Pedley T. J. The Fluid Mechanics of Large Blood Vessels, Cambridge Monographs on Mechanics and Applied Mathematics. Cambridge, Cambridge University Press, 1980, xv+446 pp. doi: $10.1017 /$ cbo9780511896996.

23. Zverev I. N., Smirnov N. N. Gazodinamika goreniia [Gas dynamics of combustion]. Moscow, Moscow State University, 1987, 307 pp. (In Russian)

Received 17/XI/2016;

received in revised form $05 / \mathrm{XII} / 2016$;

accepted 09/XII/2016. 